OPEN ACCESS

Edited by:

Deepak Gupta,

Maharaja Agrasen Institute of Technology, India

Reviewed by: Angelo D'Ambrosio, University of Turin, Italy Shankar Kathiresan,

Alagappa University, India

*Correspondence: Hany Alashwal

halashwal@uaeu.ac.ae

${ }^{\dagger}$ Data used in preparation of this article were obtained from the Alzheimer's Disease Neuroimaging Initiative (ADNI) database

(adni.loni.usc.edu). As such, the investigators within the $A D N$ contributed to the design and implementation of $A D N I$ and/or provided data but did not participate in analysis or writing of this report. A complete listing of $A D N I$ investigators can be found at: http://adni.loni.usc. edu/wp-content/uploads/ how \protect_to\protect_apply/ ADNNprotect

Acknowledgment-1protect_List.pdf

Specialty section

This article was submitted to Digital Public Health,

a section of the journal

Frontiers in Computer Science

Received: 13 April 2020 Accepted: 27 October 2020 Published: 20 November 2020

Citation:

Alashwal $H$, Diallo TMO, Tindle $R$, Moustafa AA and for the Alzheimer's Disease Neuroimaging Initiative (2020) Latent Class and Transition Analysis of

Alzheimer's Disease Data.

Front. Comput. Sci. 2:551481.

doi: 10.3389/fcomp.2020.551481

\section{Latent Class and Transition Analysis of Alzheimer's Disease Data}

\author{
Hany Alashwal ${ }^{1 *}$, Thierno M. O. Diallo ${ }^{2,3}$, Richard Tindle ${ }^{4}$, Ahmed A. Moustafa ${ }^{5,6,7}$ and \\ for the Alzheimer's Disease Neuroimaging Initiative ${ }^{\dagger}$
}

${ }^{1}$ College of Information Technology, United Arab Emirates University, Al-Ain, United Arab Emiratest, ${ }^{2}$ School of Social Science, Western Sydney University, Sydney, NSW, Australia, ${ }^{3}$ Statistiques \& M.N., Canada, ${ }^{4}$ School of Psychology, Charles Stuart University, Bathurst, NSW, Australia, ${ }^{5}$ MARCS Institute for Brain and Behaviour, Western Sydney University, Sydney, NSW, Australia, ${ }^{6}$ School of Psychology, Western Sydney University, Sydney, NSW, Australia, ${ }^{7}$ Department of Human Anatomy and Physiology, The Faculty of Health Sciences, University of Johannesburg, Johannesburg, South Africa

This study uses independent latent class analysis (LCA) and latent transition analysis (LTA) to explore accurate diagnosis and disease status change of a big Alzheimer's disease Neuroimaging Initiative (ADNI) data of 2,132 individuals over a 3-year period. The data includes clinical and neural measures of controls $(C N)$, individuals with subjective memory complains (SMC), early-onset mild cognitive impairment (EMCl), late-onset mild cognitive impairment (LMCI), and Alzheimer's disease (AD). LCA at each time point yielded 3 classes: Class 1 is mostly composed of individuals from $\mathrm{CN}, \mathrm{SMC}$, and $\mathrm{EMCl}$ groups; Class 2 represents individuals from $L M C l$ and AD groups with improved scores on memory, clinical, and neural measures; in contrast, Class 3 represents $\mathrm{LMCl}$ and from $A D$ individuals with deteriorated scores on memory, clinical, and neural measures. However, 63 individuals from Class 1 were diagnosed as AD patients. This could be misdiagnosis, as their conditional probability of belonging to Class $1(0.65)$ was higher than that of Class $2(0.27)$ and Class 3 (0.08). LTA results showed that individuals had a higher probability of staying in the same class over time with probability $>0.90$ for Class 1 and 3 and probability $>0.85$ for Class 2 . Individuals from Class 2 , however, transitioned to Class 1 from time 2 to time 3 with a probability of 0.10 . Other transition probabilities were not significant. Lastly, further analysis showed that individuals in Class 2 who moved to Class 1 have different memory, clinical, and neural measures to other individuals in the same class. We acknowledge that the proposed framework is sophisticated and time-consuming. However, given the severe neurodegenerative nature of $A D$, we argue that clinicians should prioritize an accurate diagnosis. Our findings show that LCA can provide a more accurate prediction for classifying and identifying the progression of $A D$ compared to traditional clinical cut-off measures on neuropsychological assessments.

Keywords: Alzheimer's disease, latent class analysis, latent transition analysis, neural markers, misdiagnosis

\section{INTRODUCTION}

The World Health Organization has identified Alzheimer's disease (AD) as a public health priority, with 30-35 million cases worldwide (World Health Organization, 2012). Alzheimer's disease is a chronic neurodegenerative syndrome which causes severe progressive deterioration in cognitive impairment (Alzheimer Association, 2019). Impairments include detriments in memory, learning 
ability, language, judgment, decision making, and disordered thinking (Alzheimer Association, 2019). Patients are diagnosed with $\mathrm{AD}$ after being assessed on multiple neuropsychological assessments, including memory, language functioning, personality, and behavioral changes. Assessments of specific biomarkers of $\mathrm{AD}$ are also being used to identify structural changes within specific brain regions as well as measure levels of Amyloid- $\beta$, tau, and phospho-tau (Alzheimer Association, 2019). Typically, the assessment of AD is based on clinical cut-off points for neuropsychological assessments and biomarkers. This technique allows a medical professional to identify those who have symptoms of $\mathrm{AD}$. While clinical cut-offs are important for categorizing individuals with and without $\mathrm{AD}$, it does not always contribute to our understanding of the progression of $\mathrm{AD}$ or identify individuals at risk of developing AD. Understanding the progression of $\mathrm{AD}$ is important to developing preventative interventions and earlier detection.

The Alzheimer's Disease Neuroimaging Initiative (ADNI) study has collected longitudinal data from more than 50 sites in North America on "... elderly individuals with normal cognition, mild cognitive impairment (MCI)" (Jack et al., 2008). In total, the ADNI project has collected data using 292 measurements (see http://adni.loni.usc.edu/data-samples/adnidata-inventory for a full list of items). These measurements include diagnostic assessments; neuropsychological assessments; bio-specimens; genetics; imaging_including different MRI and PET imaging techniques; demographic and medical history; and a participation record. Data were collected at $0,6,12,24$, and 36 months for participants in the normal cognition and mild cognitive impairment groups (Jack et al., 2008). However, the AD group's data were only recorded at $0,6,12$, and 24 months (Jack et al., 2008). The main aims of the ADNI project are to improve early detection and track disease progression using biomarkers and advance early intervention, prevention, and treatment.

\section{AD Diagnosis}

The assessment and diagnosis of $\mathrm{AD}$ have primarily relied on cut-off scores on neuropsychological assessments. For example, the Clinical Dementia Rating Scale (Morris, 1997) can be used to categorize individuals into differing levels of severity ranging from normal cognitive functioning, questionable cognitive impairment, questionable impairment, very mild dementia, mild dementia, moderate dementia, and severe dementia (O'Bryant et al., 2008). An advantage of utilizing categories for the Clinical Dementia Rating Scale is that it reliably identifies individuals with mild cognitive impairment (Duara et al., 2013). This allows clinicians to use the results to identify patients who are suffering from differing degrees of dementia severity. However, utilizing the scale with cut-offs does not allow health professionals to track the progression of the disease or identify at-risk patients before the presentation of symptoms.

Other neuropsychological assessments such as the Functional Activities Questionnaire (Pfeffer et al., 1982), the Alzheimer's Disease Assessment Scale (Mohs and Cohen, 1988), Clinical Dementia Rating Scale (Morris, 1997), Everyday Cognition Scale (Marshall et al., 2014), Montreal Cognitive Assessment (Nasreddine et al., 2005), the Mini-Mental State Exam (Folstein et al., 1975), and the Cognitive Change Index (Saykin et al., 2006; Rattanabannakit et al., 2016) have also been used to categorize cognitive impairment and $\mathrm{AD}$. For instance, within the ADNI, participants are classified with $\mathrm{AD}$ if they obtain a score between 20 and 26 on the mini-mental state examination; a score between 0.5 and 1.0 on the global clinical dementia rating, a score between 1.0 and 9.0 for the summed box-score for the clinical dementia rating (Shaw et al., 2009). These standardized assessments are useful for diagnosing probable $\mathrm{AD}$, with most yielding good sensitivity, specificity, and classification scores. That is, they reliably distinguish between individuals with mild cognitive impairment and $\mathrm{AD}$-making them good diagnostic tools. However, utilizing these techniques is only useful for determining probable $\mathrm{AD}$. The use of cognitive assessments only allows for a measure of current cognitive function and does not indicate if an individual may progress from mild cognitive impairment to severe cognitive impairment or $\mathrm{AD}$. In the absence of objective diagnostic assessments for $\mathrm{AD}$, a positive diagnosis is currently only determined through an autopsy (Perrin et al., 2009; Shaw et al., 2009). However, recent advances in imaging techniques (i.e., MRI and PET) and acquiring cerebral spinal fluid have allowed researchers to identify potential biomarkers of $\mathrm{AD}$ and what structural changes occur within specific brain regions (e.g., hippocampus).

Shaw et al. (2009) collected cerebral spinal fluid from elderly individuals with normal cognitive functioning, mild cognitive impairment, and mild AD (classification were determined using the mini-mental state examination and the Alzheimer's Disease Assessment Scale). The levels of Amyloid- $\beta 1$ to 42 peptide (A $\beta$ $1-42)$, total tau ( $\mathrm{t}$-tau), and tau phosphorylated ( $\mathrm{p}$-tau) were assessed to determine potential biomarkers of $\mathrm{AD}$. To gain more accurate cut-off points, models of the levels of $A \beta_{1-42}$, t-tau, and $\mathrm{p}$-tau were determined from cerebral spinal fluid samples from autopsy-confirmed $\mathrm{AD}$ cases. The results indicated that $\mathrm{A} \beta{ }_{1-42}$ showed excellent sensitivity (96.4\%) and specificity (76.9\%) with a clinical cut-off of $192 \mathrm{pg} / \mathrm{ml}$; t-Tau showed acceptable sensitivity (69.6\%) and excellent specificity (92.3\%) with a clinical cut-off of $93 \mathrm{pg} / \mathrm{ml}$, and p-tau showed acceptable sensitivity (67.9\%) and specificity $(73.1 \%)$ with a clinical cut-off of $23 \mathrm{pg} / \mathrm{ml}$. Further, the interaction between decreasing levels of $A \beta$ and increasing levels of $\mathrm{p}$-tau have recently been implicated with neuronal death, atrophy, and cognitive changes (Gomar et al., 2016; Veitch et al., 2019). These results suggest that $A \beta_{1-42}$ and p-tau are the most sensitive measures and best predictors of early diagnoses of AD.

The diagnosis of probable $\mathrm{AD}$ can also be assessed by measuring specific biomarkers (i.e., A $\beta$, t-tau, \&p-tau). However, similar to the use of neuropsychological assessments, diagnosis relies on patients exceeding a clinical threshold for the levels of each biomarker. While biomarkers of $\mathrm{AD}$ appear to reliably distinguish between those diagnosed with (i.e., sensitivity) and without $\mathrm{AD}$ (i.e., specificity), some of the measures are still below the recommended threshold of $85 \%$ for sensitivity and specificity (Ronald and National Institute on Aging Working Group, 1998; Frank et al., 2003; Shaw et al., 2009). Again, the use of clinical cut-offs only provides clinicians with a measure to differentiate between mild cognitive impairment and probable AD based on particular biomarkers. Therefore, the use of cut-off 
scores is essential for diagnosis but does not identify at-risk patients or to accurately track the progression of $\mathrm{AD}$ from mild cognitive impairment to pre-clinical $\mathrm{AD}$, probable $\mathrm{AD}$, and a final diagnosis of $\mathrm{AD}$.

To promote the early detection of $\mathrm{AD}$ and to possibly identify at-risk individuals, research should not solely rely on clinical cutoff points, which are only useful once an individual presents with neuropsychological symptoms or biomarkers associated with probable AD. Secondly, there are criticisms of using cut-off points on continuous neuropsychological assessments because patients on either side of the cut-off are likely similar (Berlin et al., 2014; Petersen et al., 2019).

\section{Latent Class Analysis}

Instead, Latent Class Analysis (LCA) can be used to identify homogeneous subgroups of individuals who are externally heterogeneous to other sub-groups (Berlin et al., 2014; Eppig et al., 2017; Mooney et al., 2018; Petersen et al., 2019; Villeneuve et al., 2019; Zammit et al., 2019a). Latent class analysis can be used to identify homogenous subgroups of $\mathrm{AD}$ based on psychological assessments (e.g., Scheltens et al., 2016; Eppig et al., 2017; Zammit et al., 2019b). For example, Scheltens et al. (2016) identified eight cognitive subtypes of $\mathrm{AD}$ within their sample of probable $\mathrm{AD}$ patients $(N=938)$. The cognitive subtypes included patients with mild-memory impairment, moderate memory impairment, mild-visuospatial-language impairment; moderate-visuospatial impairment, mild-executive functioning impairment, moderate diffuse (cognitive impairment), and severe-diffuse (cognitive impairment). The authors suggest that the identification of cognitive subtypes highlights that $\mathrm{AD}$ is a complex disease and rather than classifying individuals with $\mathrm{AD}$, we should consider differential diagnoses.

Zammit et al. (2019b) also used LCA to identify cognitive subtypes of $\mathrm{AD}$ within participants from the Rush Memory and Aging Project. Participants included in their study had no dementia at baseline; displayed signs of dementia at follow-up; were deceased at the time of the study, and had neuropathological data available. Neuropathological data were obtained from autopsies. Based on the neuropsychological outcomes at baseline (i.e., Episodic-, Semantic-, working-, and logical-memory; perceptual-and line orientation; and Perceptual Speed-Symbol Digits Modalities Test) latent class analysis was used to categories participants into 5 classes within two categories (i.e., impaired cognition and intact cognition). The impaired cognition classes included participants with mixeddomains impairment, memory-specific impairment, and frontal impairment. The intact cognition classes included participants with average cognition and superior cognition.

The aim of Zammit's (2019b) study was to identify if neuropathological evaluations at autopsy (i.e., $A \beta$, tau, hippocampal sclerosis, DNA-binding protein 43, Lewy bodies, cerebral amyloid angiopathy, atherosclerosis, and arteriolosclerosis) were predicted by the five classes of cognitive impairment and intact cognition at baseline and if the neuropathological measures differ between each class. Their results showed that baseline measurements on neuropsychological assessments were predictive of neuropathology measured at autopsy, suggesting that neuropsychological assessments are reliable for the assessment and prognosis of cognitive impairments associated with AD.

One of the main findings of Zammit et al. (2019b) study was that the biomarkers $\mathrm{A} \beta$ and Tau are strongly predictive of $\mathrm{AD}$ and can possibly be used as an early detector. Indeed, abnormal levels of $A \beta$ and Tau were strongly associated with participants within the mixed-domains class, the memory-specific class, and the frontal impairment classes. With fewer abnormalities in the average cognition class and the superior cognition class. That is, abnormal $A \beta$ and Tau were associated with impaired cognition but not intact cognition. One of the limitations of their study was that it did not account for individuals who might change classes from baseline to follow-up. For example, participants could progress from average intact cognition to memory-specific impairment. As such, the results are only capturing the class an individual belongs to at a single point in time.

Zammit et al. (2020) extended their previous work by using latent transition analysis to identify participants within the Rush Memory and Aging Project who transitioned from nonimpairment to cognitive impairment. A second aim was to compare the classification of individuals within the LTA to the clinical criteria of MCI. The results showed that across three measurements (within 12 months) cognition remained relatively stable. That is, participants did not regularly change between the five classes of impairment; identified as mixed domains impairment, memory-specific impairment, frontal Impairment, average cognition, and superior cognition. However, of the 1,924 participants, 98 individuals did change membership class from time 1 to time $2(n=62)$ and from time 2 to time $3(n=37)$. A majority of the transitions were associated with a decline in cognitive impairment at both time points. These results identified that participants who changed classes had an $86 \%$ higher risk of developing $\mathrm{AD}$ than those who did not change status. Further, their study identified 541 participants with cognitive impairment at time $2,10.5 \%$ of these participants progressed to developing dementia at time 3 . While a majority of older adults cognition remains stable, those who are experiencing some level of cognitive impairment have an elevated risk of progressing to developing dementia. The authors provide evidence that using LTA is a robust tool to identify individuals at risk of cognitive decline, identifying risk factors for interventions to target.

Zammit's (2020) study was not without limitations. Specifically, their LTA only used neuropsychological measures of episodic memory, semantic memory, working memory, and perceptual speed and orientation. With evidence suggesting that neurological biomarkers are significant and sensitive predictors of early diagnoses of AD (Shaw et al., 2009; Gomar et al., 2016; Veitch et al., 2019), it is important to identify if biological markers of $\mathrm{AD}$ can predict cognitive impairment transitions. However, their paper does highlight that LCA and LTA are at the forefront of research aiming to improve diagnostic methods and to identify individuals at risk of progressing toward $\mathrm{AD}$. Zammit et al. (2020) also note the need to validate these methods through replication of their findings, and efforts to identify homogeneous classes of cognitive impairment using other neuropsychological measures of $\mathrm{AD}$. As mentioned earlier, recent studies using 
LTA have not included biomarkers of AD. Our study adds a novel contribution to this emerging area by identifying if neuropsychological measures and neurological biomarkers of $\mathrm{AD}$ are indicators of individuals transitioning from healthy individuals to individuals with mild cognitive impairment and $\mathrm{AD}$.

\section{The Current Study}

To our knowledge, Latent Transition Analyses (LTA) has not been used to identify the neuropsychological and biomarkers associated with the progression of $\mathrm{AD}$ in terms of patients transitioning from one $\mathrm{AD}$ class to another. In the present study rather than using a set cut off point to diagnose individuals as Alzheimer's patients, Latent Class Analysis (LCA) was used to identify individuals that are more likely to develop dementia. In addition, the focus of the analysis was on the development of the individuals over time, that is, how an individual changes class membership over time. In total, the following three research aims were addressed in this study: (1) determine and describe the number of classes that best characterize individuals with respect to clinical measures and neurological biomarkers; (2) compare the classification results obtained from the LCA and the cut-off methods, to identify the misdiagnosed individuals and characterize these patients; and (3) explore the developmental course of individuals with respect to clinical and neural measures.

Below, we first describe the ADNI dataset, which we have utilized in the current study. Second, we provide details on our latent class analysis and latent transition analysis. Following that, we present the results from both latent class analysis and latent transition analysis, respectively. Finally, we discuss our results in terms of importance of our findings and clinical implications.

\section{METHOD}

\section{ADNI Dataset}

Data used in the preparation of this article were obtained from the Alzheimer's Disease Neuroimaging Initiative (ADNI) database (adni.loni.usc.edu). The ADNI was launched in 2003 as a public-private partnership, led by Principal Investigator Michael W. Weiner, MD. The primary goal of ADNI has been to test whether serial magnetic resonance imaging (MRI), positron emission tomography (PET), other biological markers, and clinical and neuropsychological assessment can be combined to measure the progression of mild cognitive impairment (MCI) and early Alzheimer's disease (AD).

The ADNI dataset includes 2,132 participants: 512 controls, and 353 with EMCI, 621 with LMCI, and 279 with SMC, and 367 AD patients. All participants were tested at 3 different times annually. In all participants, ADNI dataset includes the following measures for all participants: APOE4 = Apolipoprotein E4 gene; $\mathrm{FDG}=$ Fluorodeoxyglucose $\mathrm{CDRSB}=$ Clinical Dementia Rating Sum of Boxes; ADAS11 = Alzheimer's Disease Assessment Scale (Cognitive Subscale), 11 item version; MMSE = MiniMental State Examination; RAVimD = Rey Auditory Verbal Learning Test (Immediate word recall score); MOCA = The Montreal Cognitive Assessment; EcPtMm = Everyday Cognition-Participant Self Report (8 memory items); EcPtLg =
Everyday Cognition-Participant Self Report (9 language items); EcSPM = Everyday Cognition- Participant Study Partner Report (8 Memory items); EcSPLg = Everyday Cognition- Participant Study Partner Report (9 Language items); Hipc = Hippocampus volume; Entor = entorhinal cortex volume; Fusif $=$ fusiform gyrus volume.

\section{Statistical Analysis}

A series of Latent Class Analysis (LCA), multivariate analysis of variance, and Latent Transition Analyses (LTA) were conducted. LTA is a longitudinal extension of LCA that explores changes in class membership over time by capturing individual movements in forward and backward directions across time points. This statistical method is based on Markov chain models (Kaplan, 2008) and uses an LCA model as a measurement model.

\section{Latent Class Analysis}

Latent Class Analysis (Lazarsfeld and Henry, 1968; Clogg, 1981) was employed to empirically identify the number of classes that best characterize individuals with respect to clinical and neural measures. LCA is a mixture model that classifies participants into optimal classes on the basis of shared characteristics that distinguish members of one class from another. Furthermore, unlike traditional cluster analysis, which is based on heuristic or distance procedures (Moustafa et al., 2018; Alashwal et al., 2019), this approach is a model-based statistical method that allows the LCA solution to be replicated with an independent sample (e.g., Nylund et al., 2007).

A commonly-used strategy to determine the optimal number of classes in LCA is to estimate a series of models by progressively increasing the number of classes and comparing the models through fit statistics and tests of significance and the quality of classification across models, as well as the usefulness and the interpretability of the latent classes (e.g., Muthén and Muthén, 2000; Vermunt and Magidson, 2002). To determine the optimal number of classes for the sample, each model was evaluated using three information criteria (IC), namely, the Akaike Information Criterion (AIC; Akaike, 1987), the Bayesian Information Criterion (BIC; Schwartz, 1978), sample size adjusted BIC (SBIC; Sclove, 1987), and the Lo-MendellRubin likelihood ratio test (LMR; Lo et al., 2001). For AIC, BIC, and SBIC, a lower value indicates a better model. For the LRT, a significant $p$-value for a model with $k$ classes followed by a non-significant $p$-value for a model with $k+1$ classes indicates that the $k$ class model is the best fitting model. The indices BIC, SBIC, and LMR have been shown to identify the appropriate number of groups within finite mixture models (e.g., Diallo et al., 2016a,b; Diallo et al., 2017). Furthermore, the entropy criterion was used to examine the quality of classification across models. The normalized entropy values ranged from 0 to 1 with values $>0.80$ representing a clear assignment of individuals to latent classes. Finally, class size was also considered when determining the optimal number of latent classes. Small classes (i.e., those that contain $<5 \%$ of the sample) were considered spurious classes, as they are often associated with class over-extraction (Hipp and Bauer, 2006). 


\section{Latent Transition Analysis}

LCA can be extended to accommodate longitudinal data through LTA. LTA is a type of Markov model that studies how individuals change membership in latent classes over time. LTA links LCA variables at different time points to each other using autoregressive models. A series of multinomial logistic regression, where the latent class variable at time $t$ is regressed on the latent class variable at time $t-1$, is commonly used to estimate transitions over time in latent class membership.

\section{Analytical Steps}

The statistical analyses involved three steps. In the first step, we identified the optimal number of classes for each time point separately. In the second step, individuals were assigned to their most likely latent class (modal class assignment) and the latent class variable at time 1 is compared to the clinical diagnostic variable and misclassified patients are studied using multivariate analysis of variance. The third step involved exploring the developmental course of the patients with respect to clinical and neural measures. That is, transition probabilities were used to explore changes that had taken place in the latent classes. For this analysis, measurement invariance was assumed to ensure that the classes have the same meaning over time. Specifically, measurement model parameters were set to be equal over time. Hence, conditional item probabilities, item means, and item variances for the LCA were constrained to be equal at the three time points.

For this study, all models were estimated using a Full Information Maximum Likelihood (FIML) procedure available in Mplus 8.3 (Muthén and Muthén, 2019). FIML utilizes all available information during the estimation process and provides consistent and efficient population parameters (Enders, 2010). Furthermore, all LCA models with continuous indicators were estimated with residual variances of the outcomes constrained to be equal across classes and under local independence within classes assumption (i.e., indicators' residual covariances within classes were constrained to zero). All models with two classes or more were estimated using 500 sets of random starting values, 50 iterations for each of these sets, and the 20 best sets of random starting values associated with the highest likelihood values were retained for the final optimization stage.

\section{RESULTS}

The first aim of this study was to determine the number of classes that best characterize patients with respect to clinical and neural measures. Table 1 provides an overview of patients' characteristics with respect to clinical and neural measures. As these data showed, there was substantial variability among the patients on their clinical and neural measures. This variability supports the value of using mixture methods to assess whether the patients can be grouped into different classes based on their clinical and neural measures.

\section{Latent Class Analysis Results}

Latent class models containing 1-7 classes at each time point were fitted to the data. The model fit statistics are available in
Table 2. All LCA models converged at Time 1. The log-likelihood increased while no minimum was found for the ICs as their values decreased across the range of models considered. The LMR pointed to the three-class solution since the test of the twoclass model against the three-class model has a $p$-value of 0.003 , suggesting rejection, whereas the test of the three-class against the four-class has a $p$-value of 0.24 . Further, an examination of the LCA models indicated that the four- and five-class models each included small classes that seemed to have splintered off from larger classes in the three-class model. Therefore, a three-class model was selected at time 1 based on the fit statistics (Muthen, 2004). The three-class model resulted in a log-likelihood value of -11010.52 with 60 parameters, an AIC of 22141.04, a BIC of 22481.49, a SBIC of 22290.86, and a high entropy value of 0.89 . Moreover, the three-class solution satisfied the minimum class size required to be useful (each comprised at least $5 \%$ of the sample) and meaningful.

All models with fewer than seven classes converged at time 2 . Consequently, only model results for classes between one and six were considered for further analysis. As in time 1, log-likelihood values increased, no minimum was found for the ICs but no solution was favored by the LRT. However, similar to the results at time 1 , the results showed that the four- and five-class models each included small classes that seemed to have splintered off from larger classes in the three-class model. Hence, based on the interpretability and the usefulness of the classes, the three-class solution was also selected as the optimal number of classes at time 2. Fit indices for the three-class solution at time 2 were as follows: Log-likelihood $=-6927.78$, number of parameters $=60$, AIC $=$ $13975.57, \mathrm{BIC}=14303.75, \mathrm{SBIC}=14113.13$, and entropy $=0.82$.

Finally, all models with fewer than six classes converged at time 3, whereas models with six classes and more did not converge. Hence, only model results for classes between one and five were considered for further analysis. Similar to time 1, loglikelihood values increased, no minimum was found for the ICs, whereas the LRT selected the three-class solution. Based on the interpretability and the usefulness of the classes, the three-class solution was also selected as the optimal number of classes at time 3. Fit indices for the three-class solution at time 3 were as follows: Log-likelihood $=-6984.23$, number of parameters $=60, \mathrm{AIC}=$ $14088.45, \mathrm{BIC}=14415.74, \mathrm{SBIC}=14225.12$, and entropy $=0.81$.

\section{Explanation of Latent Class Solutions}

Here, we describe the latent class solutions at the three time points. Across the three time points, Class 1 showed a pattern of low means on CDRSB, ADAS11, EcPtMm, EcPtLg, EcSPM, EcSPLg, a pattern of high means on FDG, MMSE, RAVimD, MOCA, Hipc, Entor, Fusif, and selected category zero of with item probability $>0.65$. Class 1 is composed of $63 \%$ of the sample at time $1,59 \%$ at time 2 , and $65 \%$ at time three. In contrast, Class 3 showed a pattern of low means on FDG, MMSE, RAVimD, MOCA, Hipc, Entor, Fusif, a pattern of high means on CDRSB, ADAS11, EcPtLg, EcSPM. We, therefore, interpreted this class as the $\mathrm{AD}$ class. Class 3 is composed of $7 \%$ of the sample at time $1,10 \%$ at time 2 , and $6 \%$ at time three. Class 2 , however, showed scores that overall were between Class 1 and Class 3 . Class 2 was composed of $30 \%$ of the sample at time $1,31 \%$ at 
TABLE 1 | Descriptive statistics of the clinical and neural measures.

\begin{tabular}{|c|c|c|c|c|c|c|c|c|c|c|c|c|}
\hline \multirow[t]{2}{*}{ Variables } & \multicolumn{4}{|c|}{ Time 1} & \multicolumn{4}{|c|}{ Time 2} & \multicolumn{4}{|c|}{ Time 3} \\
\hline & $\%$ & $N$ & $\mathbf{M}$ & SD & $\%$ & $N$ & $\mathbf{M}$ & SD & $\%$ & $N$ & $\mathbf{M}$ & SD \\
\hline APOE4 & & 1,726 & & & & 1,210 & & & & 1,189 & & \\
\hline Zero & 53.10 & & & & 55.02 & & & & 50.10 & & & \\
\hline One & 37.00 & & & & 36.26 & & & & 38.01 & & & \\
\hline Two & 9.90 & & & & 8.72 & & & & 11.89 & & & \\
\hline FDG & & 735 & 1.23 & 0.15 & & 474 & 1.19 & 0.16 & & 464 & 1.18 & 0.16 \\
\hline CDRSB & & 1,990 & 1.69 & 2.39 & & 1,342 & 2.26 & 2.62 & & 1,315 & 2.12 & 2.54 \\
\hline ADAS11 & & 1,962 & 11.18 & 7.60 & & 1,335 & 12.21 & 8.47 & & 1,323 & 11.51 & 8.35 \\
\hline MMSE & & 1,978 & 27.07 & 3.32 & & 1,326 & 26.30 & 3.90 & & 1,325 & 26.49 & 3.91 \\
\hline RAVimD & & 1,956 & 36.33 & 13.47 & & 1,340 & 32.41 & 13.56 & & 1,316 & 34.01 & 13.23 \\
\hline MOCA & & 1,121 & 23.70 & 4.86 & & 1,329 & 22.56 & 5.19 & & 586 & 23.53 & 4.18 \\
\hline EcPtMm & & 1,144 & 1.98 & 0.72 & & 537 & 2.12 & 0.73 & & 589 & 2.06 & 0.73 \\
\hline EcPtLg & & 1,137 & 1.67 & 0.62 & & 545 & 1.73 & 0.65 & & 587 & 1.72 & 0.63 \\
\hline EcSPM & & 1,135 & 1.98 & 0.97 & & 557 & 2.56 & 1.03 & & 590 & 2.08 & 0.97 \\
\hline EcSPLg & & 1,138 & 1.60 & 0.80 & & 557 & 1.82 & 0.91 & & 588 & 1.67 & 0.79 \\
\hline Hipc & & 1,222 & 6676.92 & 1211.87 & & 1,177 & 6609.89 & 1243.53 & & 1,107 & 6625.67 & 1269.12 \\
\hline Entor & & 1,170 & 3437.39 & 810.34 & & 1,105 & 3397.00 & 815.24 & & 1,032 & 3414.71 & 838.77 \\
\hline Fusif & & 1,170 & 16942.23 & 2792.54 & & 1,105 & 16886.15 & 2780.76 & & 1,032 & 16877.08 & 2822.98 \\
\hline
\end{tabular}

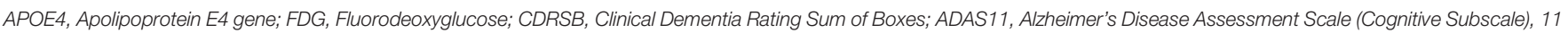

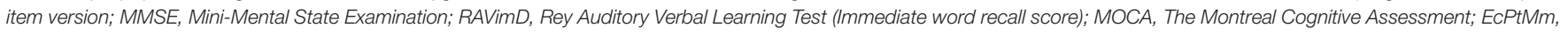

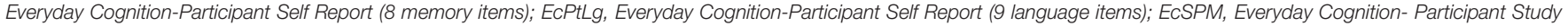

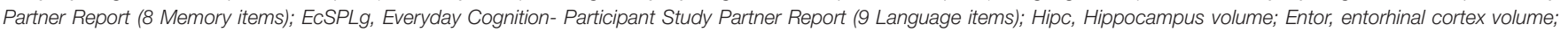
Fusif, fusiform gyrus volume. $N$ stands for number, and $M$ is for mean.

time 2, and $29 \%$ at time three. The latent class estimated for the three-class solution for the three time points are shown in Figures 1-3. Finally, cross-tabulation analysis between the LCA solution and the diagnostic variable at time 1 (Table 3 ) showed that $37.5 \%$ of the patients from Class 1 were $\mathrm{CN}, 20.10 \%$ were SMC, $19.60 \%$ were EMCI, $18.10 \%$ were LMCI, and $4.6 \%$ were AD. Similar figures were $0.5,0.9,12.90,53$, and $32.60 \%$; and 0.7 , $0,3.5,28$, and $67.80 \%$, for Class 2 and Class 3, respectively. Class 1 can be seen as composed by individuals from $\mathrm{CN}, \mathrm{SMC}$, and EMCI groups. Class 2 represents people from LMCI and from $\mathrm{AD}$ groups with improved scores on memory, clinical, and neural measures. In contrast, Class 3 represents people from LMCI and from $\mathrm{AD}$ groups with deteriorated scores on memory, clinical, and neural measures. However, 63 individuals from Class 1 were classified as $\mathrm{AD}$ individuals with the diagnosis condition.

\section{Multivariate Analysis of Variance}

Multivariate analysis of variance was used to compare the group of 63 individuals with two groups: Class 1 from the LCA without the 63 individuals and the $\mathrm{AD}$ individuals from the diagnoses variable without the 63 individuals. The results of the multivariate analysis of variance with the 13 clinical and neural measures as dependent variables resulted in multivariate F statistics of $F_{(13,1,636)}=163.85, p<0.001$, partial $\eta^{2}=0.57$. Detailed analyses revealed that group differences were significant $(0.001<p<0.01)$. The mean of the 63 individuals on the clinical and neural measures were between the mean of Class 1 without the 63 individuals and those from the $\mathrm{AD}$ individuals from the diagnoses variable without the 63 individuals (Table 4). The LCA classified the 63 individuals within Class 1 as their condition probability of belonging to this class was higher than those of belonging to the other two classes (with conditional probabilities of $0.65,0.27$, and 0.08 for Class $1,2,3$, respectively). LTA will be used to study the development of these individuals over time.

\section{Latent Transition Analysis Results}

The LTA was conducted under the measurement invariance assumption. Consequently, measurement model parameters were set to be equal over time. This ensured that the classes have the same meaning over time. Transition probabilities for the whole sample are presented in Table 5 and provide information on patient's status at time 2 given their latent status at time 1 , and patient's status at time 3 given their latent status at time 2.The results showed most individuals stayed in the same class from time 1 to time 2 but some changes in class membership for some individuals were seen from time 2 to time 3. Individuals who were in the Class 1 at time 1 had a 0.99 probability of remaining there at time 2 ( 0.88 for Class 2 and 0.98 for Class 3 , respectively). The probability (0.04) that individuals would move from the Class 2 to Class 1 by time 2 was not statistically significantly. Similarly, the probability $(0.07)$ that individuals would move from the Class 2 to the Class 1 by time 2 was not significantly different from zero. The probability $(0.02)$ that individuals would move from the Class 3 to the Class 2 by time 2 was not statistically significant either. There was, however, a 0.10 probability that individuals would transition from Class 2 to Class 
TABLE 2 | Fit statistics for model specifications at time point 1, time point 2, and time point 3.

\begin{tabular}{|c|c|c|c|c|c|c|c|}
\hline Model & Loglikelihood & \#Free parameters & AIC & BIC & SBIC & $p$ LMR & Entropy \\
\hline \multicolumn{8}{|l|}{ Time point 1} \\
\hline Two-Class & -12514.64 & 44 & 25117.28 & 25366.95 & 25227.15 & $<0.001$ & 0.88 \\
\hline Three-Class & -11010.52 & 60 & 22141.04 & 22481.49 & 22290.86 & 0.003 & 0.89 \\
\hline Four-Class & -10133.10 & 76 & 20418.21 & 20849.44 & 20607.98 & 0.24 & 0.89 \\
\hline Five-Class & -9766.25 & 92 & 19716.50 & 20238.52 & 19946.22 & 0.189 & 0.87 \\
\hline Six-Class & -9492.06 & 108 & 19200.12 & 19812.93 & 19469.80 & 0.131 & 0.83 \\
\hline Seven-Class & -9279.09 & 124 & 18806.18 & 19509.77 & 19115.81 & 0.62 & 0.80 \\
\hline \multicolumn{8}{|l|}{ Time point 2} \\
\hline Two-Class & -7936.22 & 44 & 15960.44 & 16201.10 & 16061.32 & $<0.001$ & 0.81 \\
\hline Three-Class & -6927.78 & 60 & 13975.57 & 14303.75 & 14113.13 & $<0.001$ & 0.82 \\
\hline Four-Class & -6513.27 & 76 & 13178.53 & 13594.23 & 13352.78 & 0.006 & 0.83 \\
\hline Five-Class & -6256.23 & 92 & 12696.45 & 13199.66 & 12907.38 & 0.004 & 0.75 \\
\hline Six-Class & -6087.00 & 108 & 12390.00 & 12980.73 & 12637.62 & 0.031 & 0.77 \\
\hline \multicolumn{8}{|l|}{ Time point 3} \\
\hline Two-Class & -7936.56 & 44 & 15961.11 & 16201.12 & 16061.33 & $<0.001$ & 0.79 \\
\hline Three-Class & -6984.23 & 60 & 14088.45 & 14415.74 & 14225.12 & 0.004 & 0.81 \\
\hline Four-Class & -6549.12 & 76 & 13250.23 & 13664.79 & 13423.35 & 0.514 & 0.78 \\
\hline Five-Class & -6374.21 & 92 & 12932.43 & 13434.26 & 13141.99 & 0.353 & 0.71 \\
\hline
\end{tabular}

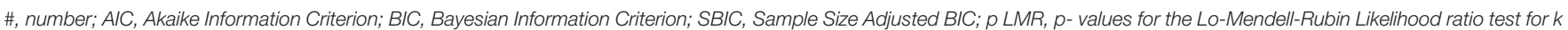

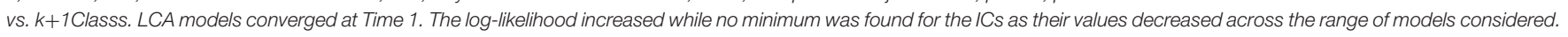

1 from time 2 to time 3. Multivariate analysis of variance showed that individuals who moved from Class 2 to Class 1 from time 2 to time 3 had significantly higher means than other individuals of Class 2 on FDG, MMSE, RAVimD, MOCA, EcPtLg, Hipc, Entor, and Fusif. But these groups of individuals had significantly lower on CDRSB, ADAS11, EcPtMm, EcSPM. No significant mean difference was found between the two groups on EcSPLang. Finally, the small number of misclassified individuals prevented us from computing the transition probabilities since LTA rely on larger sample sizes to be trustworthy. However, descriptive data show that at time 2, $42(66.7 \%)$ of the 63 individuals that were classified in Class 1 from the LCA, but AD individuals by the diagnosis variable, stayed in the Class 1 at time 2, 18 (28.60\%) moved to the Class 2 , and $3(4.80 \%)$ to the AD class. Similar figures were also found at time 3 .

\section{DISCUSSION}

The aim of this study was to use LCA to identify and describe the number of classes that best characterize CN, SMC, EMCI, LMCI, and $\mathrm{AD}$ individuals with respect to clinical and neural measures. Our second aim was to compare the classification results obtained from the LCA to more traditional cut-off methods for classifying individuals with dementia. This can help us identify and characterize potentially misdiagnosed individuals. Finally, we used LTA to investigate changes in class membership over time. Our results showed that while there was substantial variability among individuals on their clinical and neural measures, the use of LCA with mixture methods to assess grouping individuals into optimal classes yields meaningful results. We confirm that using LCA, observing model fit indices, and entropy criterion were effective for selecting the optimal number of classes of individuals. Our results identified three classes of individuals with the following characterization: $37.5 \%$ of the individuals from Class 1 were CN, 20.10\% with SMC, 19.60\% with EMCI, $18.10 \%$ with LMCI, and $4.6 \%$ (63 individuals) with AD. Similar figures were $0.5,0.9,12.90,53$, and $32.60 \%$; and $0.7,0,3.5$, 28, and $67.80 \%$, for Class 2 and Class 3, respectively. Further, our results showed that the LCA identified 63 individuals that were potentially misdiagnosed with AD. Indeed, based on the clinical and neural measures, it was deemed more probable that the misdiagnosed individuals be classified within the Class 1 instead of Class 2 or 3. Further, LTA did not show any significant change in class over time. Most individuals remained within their initial class (i.e., determined at baseline) and did not show a transition from Class 1 to Class 2, or from Class 2 to Class 3 between any time points. These results indicate that classifying individuals based on their cognitive and pathological parameters into different categories is an essential step toward understanding dementia and AD. To our knowledge, this is the first study to successfully use neuropsychological assessments and biomarkers of $\mathrm{AD}$ (e.g., Fluorodeoxyglucose, entorhinal cortex volume, and fusiform gyrus volume) to classify and predict individuals likely to transition from MCI to AD.

Similar to previous results, we identified multiple classes of cognitive impairment (Scheltens et al., 2016; Zammit et al., 2019b, 2020). For example, the results from our LCA identified 3 classes of individuals at each time point: Class 1, which is more healthy than the other classes representing 63,59, and $65 \%$ of the sample at, respectively, time 1, 2, and time 3; Class 

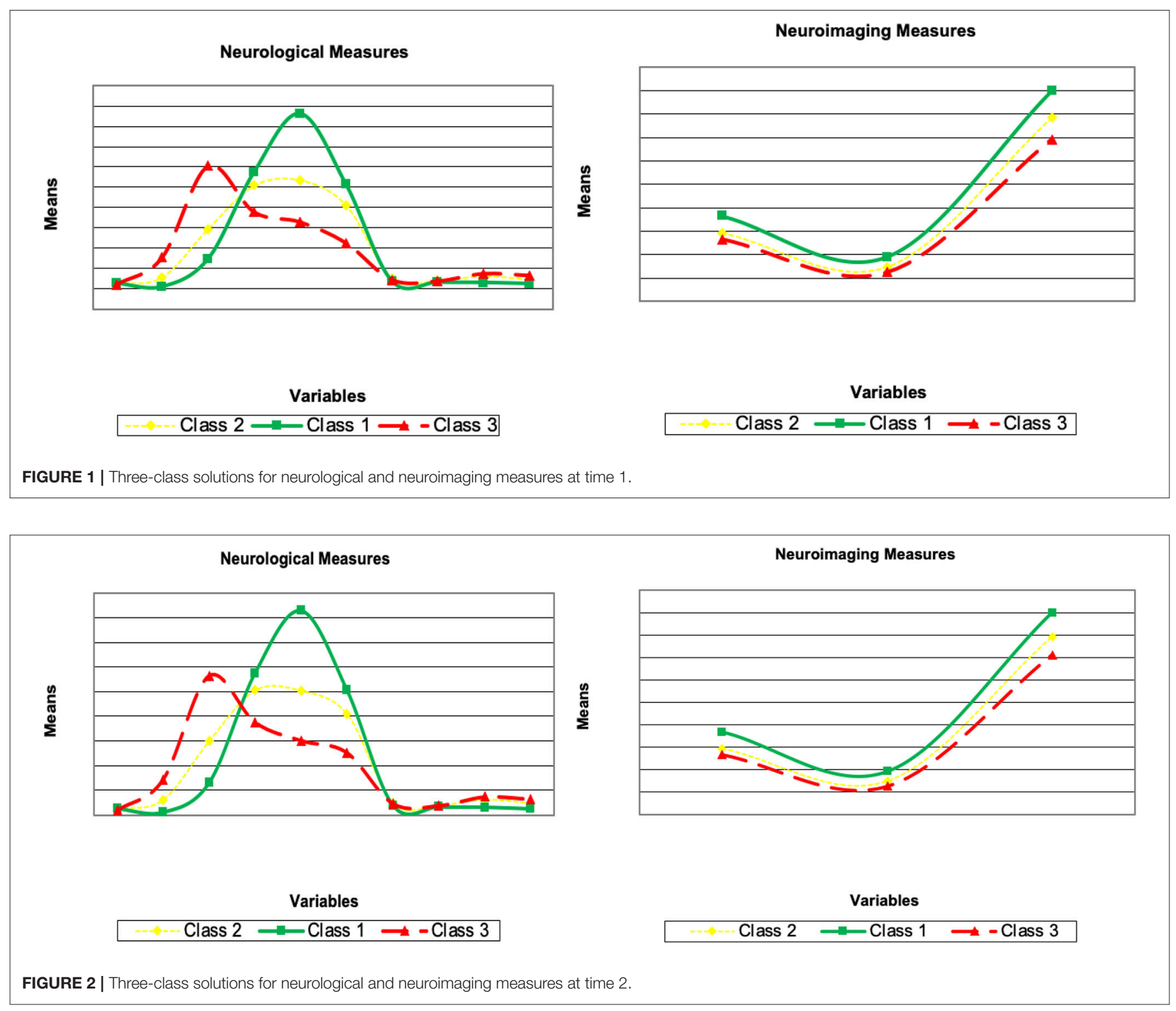

2, which lies in between Class 1 and 3, representing $\sim 30 \%$ of the sample across the three time points; and Class 3, which include the least healthy individuals, representing 7, 10, and $6 \%$ of the sample at time 1,2 , and time 3 , respectively. In other words, our LCA results reveal 3 classes that most likely match healthy individuals, individuals with MCI, and individual with AD, respectively. In comparison, Zammit et al. (2019b) classified participants into 5 classes within two categories (i.e., impaired and intact cognition) and Scheltens et al. (2016) classified participants into eight cognitive subtypes of AD. While there are differences in the number of classes identified with our results compared to others (Scheltens et al., 2016; Zammit et al., 2019b), it is important to note that the previous studies only utilized participants diagnosed with probable AD using neuropsychological assessments. Our research extends these findings by indicating that there are distinct classes of individuals who can be categorized as being healthy, experiencing MCI, and probable $\mathrm{AD}$ by using neuropsychological assessments and neurological biomarkers of $\mathrm{AD}$. This has important clinical implications as individuals can be classified as experiencing different kinds of cognitive impairment early on (i.e., at baseline) and this categorization does not change significantly across time. Consistent with recent findings (i.e., Zammit et al., 2020)., we also showed that using LCA to classify individuals with MCI or $\mathrm{AD}$ remains relatively stable over time (as indicated by LTA) and that LCA might better categorize and reduce the risk of misdiagnosis.

Our study has replicated previous findings that LCA and LTA can be used to identify homogeneous classes of cognitive impairment (Zammit et al., 2020). However, we have uniquely identified that neuropsychological measures of $\mathrm{AD}$ and the associated neurological biomarkers are indicators of an individual's class membership and can predict their likelihood to transition between the healthy class (Class 1), 


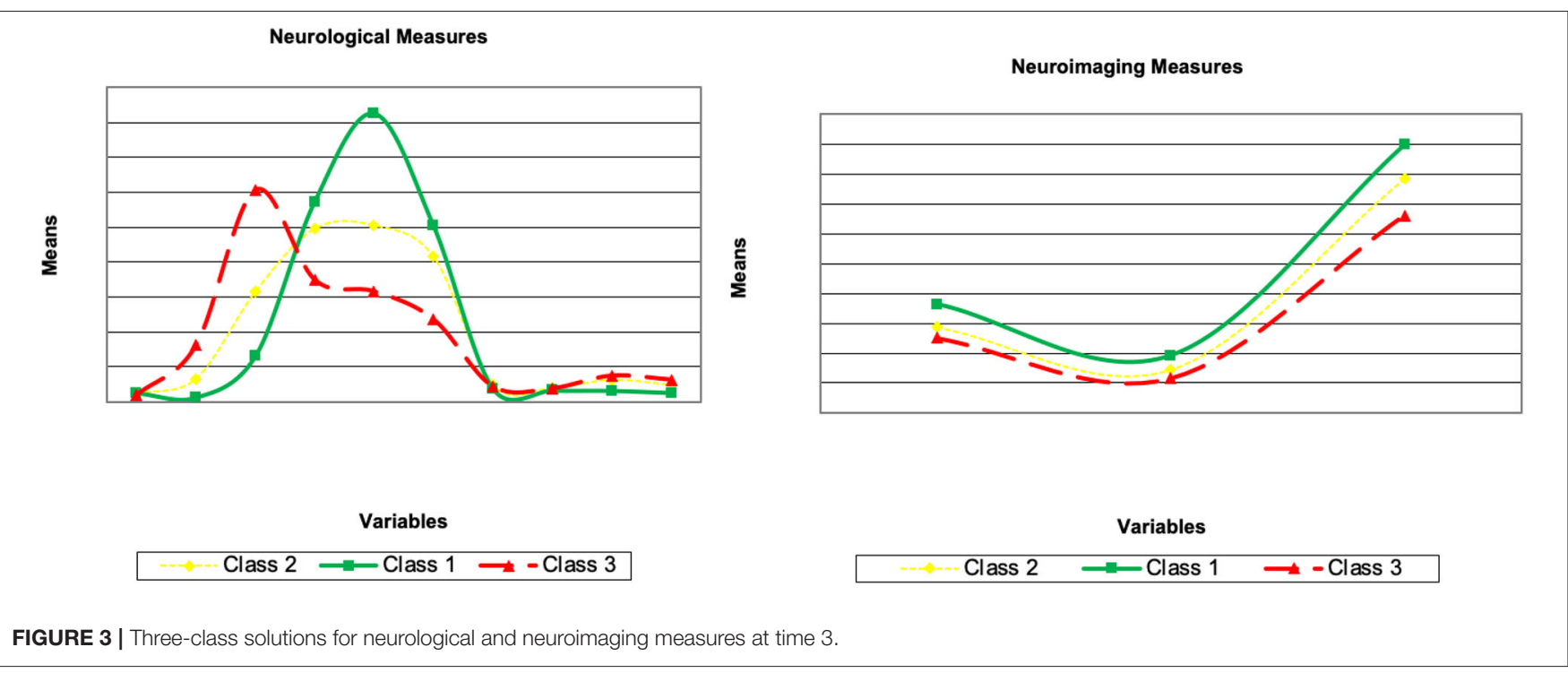
1.

\begin{tabular}{|c|c|c|c|c|c|c|}
\hline \multirow{2}{*}{$\begin{array}{l}\text { Diagnostic variable } \\
\text { at time } 1\end{array}$} & & & \multicolumn{4}{|c|}{ Latent class solution at Time 1} \\
\hline & & & Class 1 & Class 2 & Class 3 & \\
\hline & $\mathrm{CN}$ & Frequency & 508 & 3 & 1 & 512 \\
\hline & & Row \% & $99.2 \%$ & $0.6 \%$ & $0.2 \%$ & \\
\hline & & Column \% & $37.5 \%$ & $0.5 \%$ & $0.7 \%$ & \\
\hline & SMC & Frequency & 273 & 6 & 0 & 279 \\
\hline & & Row \% & $97.8 \%$ & $2.2 \%$ & $0.0 \%$ & \\
\hline & & Column \% & $20.1 \%$ & $0.9 \%$ & $0.0 \%$ & \\
\hline & $\mathrm{EMCl}$ & Frequency & 266 & 82 & 5 & 353 \\
\hline & & Row \% & $75.4 \%$ & $23.2 \%$ & $1.4 \%$ & \\
\hline & & Column \% & $19.6 \%$ & $12.9 \%$ & $3.5 \%$ & \\
\hline & $\mathrm{LMCl}$ & Frequency & 245 & 336 & 40 & 621 \\
\hline & & Row \% & $39.5 \%$ & $54.1 \%$ & $6.4 \%$ & \\
\hline & & Column \% & $18.1 \%$ & $53.0 \%$ & $28.0 \%$ & \\
\hline & $A D$ & Frequency & 63 & 207 & 97 & 367 \\
\hline & & Row \% & $17.2 \%$ & $56.4 \%$ & $26.4 \%$ & \\
\hline & & Column \% & $4.6 \%$ & $32.6 \%$ & $67.8 \%$ & \\
\hline & & & 1,355 & 634 & 143 & \\
\hline
\end{tabular}

$A D$, Alzheimer's disease; $C N$, controls; $E M C l$, early-stage mild cognitive impairment: $\mathrm{LMCl}$, late-stage mild cognitive impairment; SMC, subjective memory complains.

the MCI class (Class 2), and the AD class (Class 3). As mentioned earlier, recent studies using LTA have only utilize neuropsychological assessments of AD or MCI (e.g., Scheltens et al., 2016; Eppig et al., 2017; Zammit et al., 2019b, 2020). Our study adds a novel contribution to this emerging area by identifying that neurological biomarkers of $\mathrm{AD}$ can also be used to correctly classify individuals with MCI and $\mathrm{AD}$ and identify those at risk of transitioning from healthy cognitive function, to mild-cognitive impairment, and finally to $\mathrm{AD}$.

A comparison of the classification results obtained from the LCA with the cutoff methods at Time 1 (baseline) revealed a group of 63 misclassified individuals. This group of individuals were classified as healthy individuals by the LCA, but were classified as $\mathrm{AD}$ by using clinical cut-off scores in neuropsychological assessments. The multivariate analysis of variance revealed that the misclassified individuals' scores on the clinical and neuropsychological assessments and neurological biomarkers were bounded between the mean of the Healthy individuals (i.e., Class 1) from the LCA without the 63 individuals and those from the $\mathrm{AD}$ individuals (i.e., Class 3) from the cut-off method without the 63 individuals. However, further analysis showed that it was more probable that the misclassified participants belonged to the healthy class (i.e., Class 1) rather than the MCI class (Class 2). As LCA takes into account several clinical and neural variables, longitudinal data, as well as also considers different groups of participants, it is likely to be more accurate than standard clinical cut-off methods, which often relies on one measure and does not compare data across different groups of participants.

There is a large discrepancy between the two methods of classification (i.e., clinical assessment vs. statistical), and perhaps reinforces the criticisms of using cut-off points on continuous neuropsychological assessments. Our results show that participants either side of the cut-off are similar (Berlin et al., 2014; Petersen et al., 2019). That is, based on clinical cut-off scores, some healthy participants were identified as similar to AD individuals, which resulted in misclassification. By using LCA, we have identified homogeneous sub-groups of individuals who are externally heterogeneous to other sub-groups (Berlin et al., 2014; Eppig et al., 2017; Mooney et al., 2018; Petersen et al., 2019; Villeneuve et al., 2019; Zammit et al., 2019a). For example, the healthy class (i.e., Class 1) is externally heterogeneous compared to the MCI class (Class 2) and the AD class (i.e., 
TABLE 4 | Analysis of variance results with mean and standard deviations.

\begin{tabular}{|c|c|c|c|c|c|}
\hline & The 63 patients (MD) & Diagnostics minus the 63 (DG) & Healthy minus the $63(\mathrm{HT})$ & $d$ & Contrasts \\
\hline \multicolumn{6}{|l|}{ Measures } \\
\hline FDG $M(S D)$ & $1.26(0.13)$ & $1.06(0.15)$ & $1.29(0.12)$ & $0.29^{\star \star \star}$ & $\mathrm{MD}=\mathrm{HT}>\mathrm{DG}$ \\
\hline ADAS11M (SD) & $1.14(0.61)$ & $2.16(0.90)$ & $0.75(0.39)$ & $0.52^{\star \star \star}$ & $\mathrm{DG}>\mathrm{MD}>\mathrm{HT}$ \\
\hline MMSEM (SD) & $2.70(0.28)$ & $2.24(0.34)$ & $2.87(0.15)$ & $0.52^{\star \star \star}$ & $\mathrm{HT}>\mathrm{MD}>\mathrm{DG}$ \\
\hline EcPtMmM (SD) & $2.09(0.74)$ & $2.27(0.70)$ & $1.86(0.66)$ & $0.05^{\star \star \star}$ & $\mathrm{DG}>\mathrm{MD}>\mathrm{HT}$ \\
\hline $\operatorname{EcPtLgM}(S D)$ & $1.77(0.66)$ & $1.84(0.66)$ & $1.60(0.57)$ & $0.02^{\star \star \star}$ & $\mathrm{DG}>\mathrm{MD}>\mathrm{HT}$ \\
\hline $\operatorname{EcSPM} M(S D)$ & $2.08(0.83)$ & $2.99(0.76)$ & $1.59(0.61)$ & $0.41^{\star \star *}$ & $\mathrm{DG}>\mathrm{MD}>\mathrm{HT}$ \\
\hline $\operatorname{EcSPLg} M(S D)$ & $1.70(0.69)$ & $2.36(0.79)$ & $1.31(0.46)$ & $0.36^{\star \star \star}$ & $\mathrm{DG}>\mathrm{MD}>\mathrm{HT}$ \\
\hline HipcM $(S D)$ & $0.70(0.12)$ & $0.56(0.11)$ & $0.72(0.10)$ & $0.28^{\star \star \star}$ & $\mathrm{MD}=\mathrm{HT}>\mathrm{DG}$ \\
\hline
\end{tabular}

${ }^{* \star *} p<0.001$. All variables are defined in the text and in the captions of prior tables.

TABLE 5 | Transition probabilities from Time 1 to Time 2 and from Time 2 to Time 3.

\begin{tabular}{|c|c|c|c|c|c|c|c|c|c|}
\hline & & \multicolumn{3}{|c|}{ T2 } & & & \multicolumn{3}{|c|}{ T3 } \\
\hline & & Class 1 & Class 2 & Class 3 & & & Class 1 & Class 2 & Class 3 \\
\hline \multirow[t]{3}{*}{ T1 } & Class 1 & $0.99 a^{*}$ & 0.01 & 0.00 & T2 & Class 1 & $0.93^{\star}$ & 0.07 & 0.00 \\
\hline & Class 2 & $0.04^{b}$ & $0.88^{\star}$ & 0.07 & & Class 2 & $0.10^{*}$ & $0.85^{\star}$ & 0.05 \\
\hline & Class 3 & 0.00 & 0.02 & $0.98^{*}$ & & Class 3 & 0.00 & 0.09 & $0.91^{*}$ \\
\hline
\end{tabular}

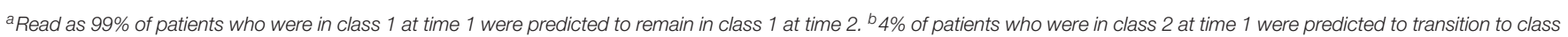
1 at time 2.

*probabilities significantly different from 0 at $p<0.05$.

Class 3). Conversely, the AD class (i.e., Class 3) is relatively more homogenous (i.e., after identifying misdiagnosed individuals) and externally heterogeneous compared to the healthy class and MCI class.

The LTA results were used to examine the individuals' transition probabilities with respect to clinical assessments, neurological measures, and neurological biomarkers to explore changes in the latent classes over time. The LTA results showed that individuals in the Healthy (i.e., Class 1) and AD (i.e., Class 3) classes were stable (with a probability $>0.90$ of staying in the same class over time). The results show that the transition from Healthy to AD classes was non-existent. This is consistent with the nature of the $\mathrm{AD}$ as a progressive disease. Individuals at early stages do not exhibit symptoms of $\mathrm{AD}$. However, there is an insignificant probability for the individuals in the healthy class to transition to the MCI class ( 0.01 from time 1 to time 2 and 0.07 from time 2 to time 3). Furthermore, the results confirm the nature of $\mathrm{AD}$ as a neurodegenerative disease (Alzheimer Association, 2019). For example, at no time-point did individuals who are in the $\mathrm{AD}$ class show cognitive improvement by transitioning to the Healthy class or MCI class.

In contrast, individuals from the MCI class have non-zero probabilities moving to other classes over time. However, only the probability of transitioning to the Healthy class from time
2 to time 3 was significant (with a probability of 0.10 ). That is, some individuals classified within the MCI class (Class 2) showed cognitive improvement from time 2 to time 3 . This further emphasizes the differences between MCI and AD. While $\mathrm{AD}$ is a regressive disease that does not allow cognitive improvements, MCI is not necessarily degenerative. As such, we should be cautious about suggesting individuals with MCI are on a progression toward AD. As we only observed movement from the MCI class into the healthy class, it is less probable that individuals with MCI will progress into AD. Therefore, based on our findings, any transition from the MCI class is likely to resemble cognitive improvement rather than decline. It is worth noting that we did not identify the characteristics that predict the movement of individuals from the MCI class. Further research is needed to investigate possible factors that may contribute to this movement, either to the Healthy or AD class.

\section{LIMITATIONS}

It is important to note that one main advantage of traditional rule-based diagnostic methods (as often used by most clinicians and doctors) is easy utilization in the everyday clinical setting. However, our methods used here are more complex and 
require applying analytical and statistical method to be able to reach a more robust diagnosis. Accordingly, because of its complexity (e.g., conducting the analysis and interpretations of results), it is expected that latent class analysis methods may not be widely used. However, we also agree that it is exactly LCA complexity over the traditional discrete diagnostic methods (e.g., surveys) that allow it to be a better predictor of class membership (e.g., an individual is healthy, has mild cognitive impairment, or has Alzheimer's disease). This is due to the fact that rule-based diagnostic methods are inherently additive, as they rely on discrete methods. However, LCA is a multivariate approach that attempts to find complex joint probability distributions that create a richer risk profile which is difficult to define using discrete rule-based decision tools and diagnostic methods.

\section{CONCLUSION}

In conclusion, this study demonstrated that latent class analysis can be used to classify participants within the ADNI project into three distinct classes: Healthy, MCI, or $\mathrm{AD}$. We argue that LCA is a more suitable method for classifying individuals with SMC, MCI, and $\mathrm{AD}$ rather than using clinical cut-off measures. This is due to LCA's ability to create internally homogenous and externally heterogeneous sub-groups. This technique might help reduce the number of misclassifications of individuals incorrectly diagnosed with probable $\mathrm{AD}$-as demonstrated by the misclassified individuals in our study. By using latent transition analysis, we showed that individuals classified as healthy or with $\mathrm{AD}$ had a high probability of staying in the same class over time. However, it was more probable for individuals to transition from the MCI class to the healthy class. Our results emphasize that $\mathrm{AD}$ is a neurodegenerative syndrome, with individuals within the $\mathrm{AD}$ class showing no evidence of cognitive improvement over time. However, individuals with MCI can show improvement over time. Therefore, we argue that LCA can be used to differentiate between individuals with $\mathrm{AD}$ and that this diagnosis remains stable across time and produces fewer misdiagnoses than using clinical cut-offs. Robust methods should be used to accurately diagnose patients and to identify individuals at a highest risk of developing AD. While using cut-off scores using traditional discrete diagnostic methods are quicker, our study has shown that LCA can provide a more accurate prediction for classifying individuals with SMC, MCI, and AD. While the time requirement to conduct LCA is burdensome, ensuring an accurate diagnosis for patients should be a prioritized. Especially given the severity and neurodegenerative nature of $\mathrm{AD}$ (Alzheimer Association, 2019). Using LCA and LTA can provide more accurate diagnoses and improve the outcomes for patients.

\section{REFERENCES}

Akaike, H. (1987). Factor analysis and AIC. Psychometrika 52, 317-332. doi: 10.1007/BF02294359
Clinicians should consider alternative diagnostic methods for $\mathrm{AD}$ instead of relying solely on the clinical cut-off measures on neuropsychological assessments.

\section{DATA AVAILABILITY STATEMENT}

Publicly available datasets were analyzed in this study. This data can be found here: http://adni.loni.usc.edu.

\section{AUTHOR CONTRIBUTIONS}

All authors contributed to data analysis, writing, and editing the manuscript.

\section{FUNDING}

This work received financial support from the United Arab Emirates University (grant no. CIT 31T129).

\section{ACKNOWLEDGMENTS}

Data collection and sharing for this project was funded by the Alzheimer's Disease Neuroimaging Initiative (ADNI) (National Institutes of Health Grant U01 AG024904) and DOD ADNI (Department of Defense award number W81XWH-12-2-0012). ADNI was funded by the National Institute on Aging, the National Institute of Biomedical Imaging and Bioengineering, and through generous contributions from the following: AbbVie, Alzheimer's Association; Alzheimer's Drug Discovery Foundation; Araclon Biotech; BioClinica, Inc.; Biogen; BristolMyers Squibb Company; CereSpir, Inc.; Cogstate; Eisai Inc.; Elan Pharmaceuticals, Inc.; Eli Lilly and Company; EuroImmun; F. Hoffmann-La Roche Ltd and its affiliated company Genentech, Inc.; Fujirebio; GE Healthcare; IXICO Ltd.; Janssen Alzheimer Immunotherapy Research \& Development, LLC.; Johnson \& Johnson Pharmaceutical Research \& Development LLC.; Lumosity; Lundbeck; Merck \& Co., Inc.; Meso Scale Diagnostics, LLC.; NeuroRx Research; Neurotrack Technologies; Novartis Pharmaceuticals Corporation; Pfizer Inc.; Piramal Imaging; Servier; Takeda Pharmaceutical Company; and Transition Therapeutics. The Canadian Institutes of Health Research is providing funds to support ADNI clinical sites in Canada. Private sector contributions are facilitated by the Foundation for the National Institutes of Health (www.fnih.org). The grantee organization is the Northern California Institute for Research and Education, and the study is coordinated by the Alzheimer's Therapeutic Research Institute at the University of Southern California. ADNI data are disseminated by the Laboratory for Neuro Imaging at the University of Southern California. 
Alzheimer Association (2019). Early Signs and Symptoms of Alzheimer's. Alzheimer's and Dementia, 1-88. Retrieved from https://www.alz.org/ alzheimers-dementia/10_signs

Berlin, K. S., Williams, N. A., and Parra, G. R. (2014). An introduction to latent variable mixture modeling (Part 1): overview and cross-sectional latent class and latent profile analyses. J. Pediatr. Psychol. 39, 174-187. doi: 10.1093/jpepsy/jst084

Clogg, C. C. (1981). "New developments in latent structure analysis," in Factor Analysis and Measurement in Sociological Research. eds D. J. Jackson and E. F. Borgatta (London: Sage), 77-108.

Diallo, T. M. O., Morin, A. J. S., and Lu, H. (2016a). Impact of misspecifications of the latent variance-covariance and residual matrices on the class enumeration accuracy of growth mixture models. Struct. Equat. Model. 23, 507-531. doi: $10.1080 / 10705511.2016 .1169188$

Diallo, T. M. O., Morin, A. J. S., and Lu, H. (2016b). Performance of growth mixture models in the presence of time-varying covariates. Behav. Res. Methods 49, 1951-1965. doi: 10.3758/s13428-016-0823-0

Diallo, T. M. O., Morin, A. J. S., and Lu, H. (2017). The impact of total and partial inclusion or exclusion of active and inactive time invariant covariates in growth mixture models. Psychol. Methods 22, 166-190. doi: 10.1037/met0000084

Duara, R., Loewenstein, D. A., Shen, Q., Barker, W., Varon, D., Greig, M. T., et al. (2013). The utility of age-specific cut-offs for visual rating of medial temporal atrophy in classifying Alzheimer's disease, $\mathrm{MCI}$ and cognitively normal elderly subjects. Front. Aging Neurosci. 5:47. doi: 10.3389/fnagi.2013.00047

Enders, C. K. (2010). Applied Missing Data Analysis. New York, NY: Guilford Press.

Eppig, J. S., Edmonds, E. C., Campbell, L., Sanderson-Cimino, M., DelanoWood, L., and Bondi, M. W. (2017). Statistically derived subtypes and associations with cerebrospinal fluid and genetic biomarkers in mild cognitive impairment: a latent profile analysis. J. Int. Neuropsychol. Soc. 23, 564-576. doi: $10.1017 /$ S135561771700039X

Folstein, M. F., Folstein, S. E., and McHugh, P. R. (1975). "Mini-mental state": a practical method for grading the cognitive state of patients for the clinician. $J$. Psychiatr Res. 12, 189-198. doi: 10.1016/0022-3956(75)90026-6

Frank, R. A., Galasko, D., Hampel, H., Hardy, J., de Leon, M. J., Mehta, P. D., et al. (2003). Biological markers for therapeutic trials in Alzheimer's disease-proceedings of the biological measures working group: NIA Initiative on Neuro-imaging in Alzheimer's disease. Neurobiol. Aging 24, 521-536. doi: 10.1016/S0197-4580(03)00002-2

Gomar, J. J., Conejero-Goldberg, C., Huey, E. D., Davies, P., Goldberg, T. E., and Alzheimer's Disease Neuroimaging Initiative. (2016). Lack of neural compensatory mechanisms of BDNF val66met met carriers and APOE E4 carriers in healthy aging, mild cognitive impairment, and Alzheimer's disease. Neurobiol. Aging 39, 165-173. doi: 10.1016/j.neurobiolaging.2015.12.004

Hipp, J. R., and Bauer, D. J. (2006). Local solutions in the estimation of growth mixture models. Psychol. Methods 11, 36-53. doi: 10.1037/1082-989X.11.1.36

Jack, C. R., Bernstein, M. A., Fox, N. C., Thompson, P., Alexander, G., Harvey, D., et al. (2008). The Alzheimer's Disease Neuroimaging Initiative (ADNI): MRI methods. J. Magn. Reson. Imaging 27, 685-691. doi: 10.1002/jmri.21049

Kaplan, D. (2008). An overview of markov chain methods for study of stage-sequential developmental processes. Dev. Psychol. 44, 457-467. doi: 10.1037/0012-1649.44.2.457

Lazarsfeld, P. F., and Henry, N. W. (1968). Latent Structure Analysis. Boston, MA: Houghton Mill.

Lo, Y., Mendell, N., and Rubin, D. (2001). Testing the number of components in a normal mixture. Biometrika 88, 767-778. doi: 10.1093/biomet/88.3.767

Marshall, G.A,. Zoller, A. S., Kelly, K. E., Amariglio, R. E., Locascio, J. J., Johnson, K. A., et al. (2014). Everyday cognition scale items that best discriminate between and predict progression from clinically normal to mild cognitive impairment. Curr. Alzheimer Res. 11, 853-861. doi: $10.2174 / 1567205011666141001120903$

Mohs, R. C., and Cohen, L. (1988). Alzheimer's disease assessment scale (ADAS). Psychopharmacol. Bull. 24, 627-628.

Mooney, S. J., Joshi, S., Cerdá, M., Kennedy, G. J., Beard, J. R., and Rundle, A. G. (2018). Longitudinal patterns of physical activity among older adults: a latent transition analysis. Am. J. Epidemiol. 187, 1549-1558. doi: 10.1093/aje/kwy027

Morris, J. C. (1997). Clinical dementia rating: a reliable and valid diagnostic and staging measure for dementia of the Alzheimer type. Int. Psychogeriatr. 9, 173-176. doi: 10.1017/S1041610297 004870

Moustafa, A. A., Diallo, T., Amoroso, N., Zaki, N., Hassan, M., and Alashwal, H. (2018). Applying big data methods to understanding human behavior and health. Front. Comput. Neurosci. 12:84. doi: 10.3389/fncom.2018.00084

Muthen, B. (2004). "Latent variable analysis: growth mixture modeling and related techniques for longitudinal data," in Handbook of Quantitative Methodology for the Social Sciences, ed D. Kaplan. (Thousand Oaks, CA: Sage), 345-368.

Muthén, B. O., and Muthén, L. K. (2000). Integrating person-centered and variable-centered analyses: growth mixture modeling with latent trajectory classes. Alcohol. Clin. Exp. Res. 24, 882-891. doi: 10.1111/j.1530-0277.2000.tb02070.x

Muthén, L. K., and Muthén, B. O. (2019). Mplus User's Guide. New York, NY: Author.

Nasreddine, Z. S., Phillips, N. A., Bédirian, V., Charbonneau, S., Whitehead, V., Collin, I., et al. (2005). The montreal cognitive assessment, MoCA: a brief screening tool for mild cognitive impairment. J. Am. Geriatr. Soc. 53, 695-699. doi: 10.1111/j.1532-5415.2005.53221.x

Nylund, K. L., Asparouhov, T., and Muthén, B. O. (2007). Deciding on the number of classes in latent class analysis and growth mixture modeling: a monte carlo simulation study. Struc. Equat. Model. 14, 535-569. doi: 10.1080/10705510701575396

O’Bryant, S. E., Waring, S. C., Cullum, C. M., Hall, J., Lacritz, L., Massman, P. J., et al. (2008). Staging dementia using clinical dementia rating scale sum of boxes scores: a texas Alzheimer's research consortium study. Arch. Neurol. 65, 1091-1095. doi: 10.1001/archneur.65.8.1091

Perrin, R. J., Fagan, A. M., and Holtzman, D. M. (2009). Multimodal techniques for diagnosis and prognosis of Alzheimer's disease. Nature 461, 916-922. doi: 10.1038/nature08538

Petersen, K. J., Qualter, P., and Humphrey, N. (2019). The application of latent class analysis for investigating population child mental health: a systematic review. Front. Psychol. 10:1214. doi: 10.3389/fpsyg.2019.01214

Pfeffer, R. I., Kurosaki, T. T., Harrah C. H. Jr, Chance, J. M., and Filos, S. (1982). Measurement of functional activities in older adults in the community. $J$. Gerontol. 37, 323-329. doi: 10.1093/geronj/37.3.323

Rattanabannakit, C., Risacher, S. L., Gao, S., Lane, K. A., Brown, S. A., McDonald, B. C., et al. (2016). The cognitive change index as a measure of self and informant perception of cognitive decline: relation to neuropsychological tests. J. Alzheimers Dis. 51, 1145-1155. doi: 10.3233/JAD-150729

Ronald, T., and National Institute on Aging Working Group (1998). Consensus report of the working group on:"Molecular and biochemical markers of Alzheimer's disease". Neurobiol. Aging 19, 109-116. doi: 10.1016/S0197-4580(98)00022-0

Saykin, A. J., Wishart, H. A., Rabin, L. A., Santulli, R. B., Flashman, L. A., West, J. D., et al. (2006). Older adults with cognitive complaints show brain atrophy similar to that of amnestic MCI. Neurology 67, 834-842. doi: 10.1212/01.wnl.0000234032.77541.a2

Scheltens, N. M. E., Galindo-Garre, F., Pijnenburg, Y. A. L., Van Der Vlies, A. E., Smits, L. L., Koene, T., et al. (2016). The identification of cognitive subtypes in Alzheimer's disease dementia using latent class analysis. J. Neurol. Neurosurg. Psychiatry 87, 235-243. doi: 10.1136/jnnp-2014-309582

Schwartz, G. (1978). Estimating the dimensions of a model. Ann. Stat. 6, 461-464. doi: 10.1214/aos/1176344136

Sclove, L. (1987). Application of model-selection criteria to some problems in multivariate analysis. Psychometrika 52, 333-343. doi: 10.1007/BF02294360

Shaw, L. M., Vanderstichele, H., Knapik-Czajka, M., Clark, C. M., Aisen, P. S., Petersen, R. C., et al. (2009). Cerebrospinal fluid biomarker signature in alzheimer's disease neuroimaging initiative subjects. Ann. Neurol. 65, 403-413. doi: 10.1002/ana.21610

Veitch, D. P., Weiner, M. W., Aisen, P. S., Beckett, L. A., Cairns, N. J., Green, R. C., et al. (2019). Understanding disease progression and improving Alzheimer's disease clinical trials: recent highlights from the Alzheimer's disease neuroimaging initiative. Alzheimers Dement. 15, 106-152. doi: $10.1016 /$ j.jalz.2018.08.005

Vermunt, J. K., and Magidson, J. (2002). "Latent class cluster analysis," in Applied Latent Class Analysis, eds J. A. Hagenaars and A. L. McCutcheon (Cambridge: Cambridge University Press), 89-106. doi: 10.1017/CBO9780511499 531.004 
Villeneuve, S. C., Houot, M., Cacciamani, F., Verrijp, M., Dubois, B., Sikkes, S., et al. (2019). Latent class analysis identifies functional decline with Amsterdam IADL in preclinical Alzheimer's disease. Alzheimers Dement. 5, 553-562. doi: $10.1016 /$ j.trci.2019.08.009

World Health Organization (2012). Dementia: A Public Health Priority. Dementia.

Zammit, A. R., Bennett, D. A., Hall, C. B., Lipton, R. B., Katz, M. J., and MunizTerrera, G. (2020). A Latent transition analysis model to assess change in cognitive states over three occasions: results from the rush memory and aging project. J. Alzheimers Dis. 73, 1063-1073. doi: 10.3233/JAD-190778

Zammit, A. R., Hall, C. B., Bennett, D. A., Ezzati, A., Katz, M. J., Munizterrera, G., et al. (2019a). Neuropsychological latent classes at enrollment and postmortem neuropathology. Alzheimers Dement. 15, 1195-1207. doi: 10.1016/j.jalz.2019.05.012

Zammit, A. R., Terrera, G. M., Hall, C. B., Katz, M. J., Bennett, D. A., Ezzati, A., et al. (2019b). A latent transition analysis model to assess change in cognitive states and predict incident dementia: results from the rush memory and aging project. Alzheimers Dement. 15:P445. doi: 10.1016/j.jalz.2019. 06.1067

Conflict of Interest: The authors declare that the research was conducted in the absence of any commercial or financial relationships that could be construed as a potential conflict of interest.

Copyright (c) 2020 Alashwal, Diallo, Tindle, Moustafa and for the Alzheimer's Disease Neuroimaging Initiative. This is an open-access article distributed under the terms of the Creative Commons Attribution License (CC BY). The use, distribution or reproduction in other forums is permitted, provided the original author(s) and the copyright owner(s) are credited and that the original publication in this journal is cited, in accordance with accepted academic practice. No use, distribution or reproduction is permitted which does not comply with these terms. 